\title{
ST-elevation versus non-ST-elevation myocardial infarction after combined use of statin with renin-angiotensin system inhibitor: Data from the Korea Acute Myocardial Infarction Registry
}

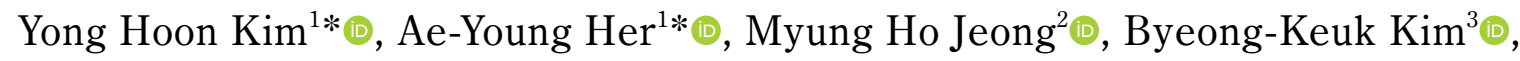

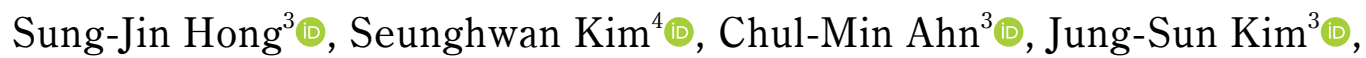 \\ Young-Guk $\mathrm{Ko}^{3}$ (D) Donghoon $\mathrm{Choi}^{3}$ (D), Myeong-Ki Hong ${ }^{3}$, , Yangsoo Jang ${ }^{3}$ (1) \\ ${ }^{1}$ Division of Cardiology, Department of Internal Medicine, Kangwon National University \\ School of Medicine, Chuncheon, Republic of Korea \\ ${ }^{2}$ Department of Cardiology, Cardiovascular Center, Chonnam National University Hospital, \\ Gwangju, Republic of Korea \\ ${ }^{3}$ Division of Cardiology, Severance Cardiovascular Hospital, Yonsei University \\ College of Medicine, Seoul, Republic of Korea \\ ${ }^{4}$ Division of Cardiology, Inje University College of Medicine, Haeundae Paik Hospital, \\ Busan, Republic of Korea
}

\begin{abstract}
Background: Limited data are available comparing the combined effects of statins and renin-angiotensin system inhibitor (RASI) between patients with ST-segment elevation myocardial infarction (STEMI) and those with non-STEMI (NSTEMI). We compared the effects of statins combined with RASI in STEMI and NSTEMI patients after stent implantation during a 2-year follow-up period.

Methods: A total of 21,890 acute myocardial infarction (AMI) patients who underwent successful stent implantation and who received statins with RASI were enrolled. They were separated into the STEMI group $(n=12,490)$ and the NSTEMI group $(n=9400)$. The major clinical endpoint was the occurrence of major adverse cardiac events (MACEs) defined as all-cause death, recurrent myocardial infarction (Re-MI), and any repeat revascularization.

Results: Two propensity score-matched groups (5891 pairs, $n=11,782, C$-statistic $=0.821$ ) were generated. Even though the cumulative incidences of MACE, Re-MI, total repeat revascularization were similar between the two groups, the cumulative incidences of all-cause death (hazard ratio [HR] 1.407; 95\% confidence interval [CI] 1.106-1.790; $p=0.005)$ and cardiac death (HR 1.311; 95\% CI $0.983-1.749 ; p=0.046$ ) were significantly higher in the NSTEMI group.

Conclusions: In this study, statin with RASI combination therapy was more beneficial to the STEMI patients than to the NSTEMI patients in reducing all-cause death and cardiac death. (Cardiol J 2022; 29, 4: 647-659)

Key words: non-ST-segment elevation myocardial infarction, renin-angiotensin system, statin, ST-segment elevation myocardial infarction
\end{abstract}

Address for correspondence: Yong Hoon Kim, MD, PhD, Division of Cardiology, Department of Internal Medicine, Kangwon National University School of Medicine, 24289, 156 Baengnyeong Road, Chuncheon City, Gangwon Province, Republic of Korea, tel: +82-33-258-9455, fax: +82-33-258-2455, e-mail: yhkim02@kangwon.ac.kr

Received: 18.06.2020 Accepted: 15.01.2021 Early publication date: 20.01.2021

*The first two authors (Yong Hoon Kim and Ae-Young Her) contributed equally to this work.

This article is available in open access under Creative Common Attribution-Non-Commercial-No Derivatives 4.0 International (CC BY-NC-ND 4.0) license, allowing to download articles and share them with others as long as they credit the authors and the publisher, but without permission to change them in any way or use them commercially. 


\section{Introduction}

Intensive statin therapy has produced greater reductions in the risks of cardiovascular death, non-fatal myocardial infarction (MI), ischemic stroke, and coronary revascularization than less intensive statin therapy in patients with acute coronary syndrome [1-3]. Moreover, stains are recommended for all acute MI (AMI) patients, regardless of cholesterol concentration at presentation [4-6]. More recently, Kim et al. [7] reported that statin therapy was more effective in reducing the cumulative risks of major adverse cardiac events (MACEs), all-cause death, and target vessel revascularization (TVR) in a ST-segment elevation myocardial infarction (STEMI) group than in a non-STEMI (NSTEMI) group in Korean patients with AMI after successful drug-eluting stent (DES) implantation. Current guidelines recommended that angiotensin-converting enzyme inhibitors (ACEIs) should be prescribed within the first 24 hours for all AMI patients with left ventricular (LV) systolic dysfunction, unless contraindicated. Furthermore, the patients who do not tolerate ACEIs should be given angiotensin receptor blockers (ARBs) $[5,6,8,9]$. A previous report [10] showed that the mortality reduction capability of renin-angiotensin system inhibitors (RASIs) was more prominent in STEMI patients compared with NSTEMI patients. Hence, combination therapy with statins and RASIs may be an important treatment modality in patients with hypertension, hypercholesterolemia, diabetes, metabolic syndrome, or obesity, to reduce or prevent cardiovascular disease $[11,12]$. Nevertheless, the data concerning long-term clinical outcomes of statin with RASI combination therapy in patients with STEMI and NSTEMI after stent implantation are limited. Therefore, we compared the effects of statins combined with RASI in STEMI and NSTEMI patients after successful percutaneous coronary intervention (PCI) over a 2-year follow-up period.

\section{Methods}

\section{Study design and population}

The study population of this non-randomized, multicenter, observational, retrospective cohort study was obtained from the Korea AMI Registry (KAMIR). KAMIR was designed to capture real-world treatment practices and the short- and long-term outcomes of AMI patients; to evaluate the current epidemiology and analyze the prognostic factors of AMI; and to improve the long-term prognosis of the individual patients.
Eligible patients were $\geq 18$ years of age at the time of hospital admission [13]. A total of 45,863 AMI patients who underwent successful stent implantation from November 2005 to June 2015 were evaluated. This study protocol was approved by the ethics committee at each participating center, and informed consent was obtained from all individual participants prior to enrollment. These processes were conducted according to the ethical guidelines of the 1975 Declaration of Helsinki. The exclusion criteria were as follows: (1) incomplete laboratory results ( $\mathrm{n}=10,506,22.9 \%)$; (2) lost to follow-up ( $\mathrm{n}=2562,5.6 \%)$; (3) statin and RASI had not been prescribed ( $\mathrm{n}=2392,5.2 \%)$; (4) statin only prescribed $(\mathrm{n}=4409,9.6 \%)$; and (5) RASI only prescribed $(\mathrm{n}=4185,9.1 \%)$. Finally, a total of 21,890 AMI patients who underwent successful stent implantation and who had been prescribed both statin and RASI were enrolled. Among these, 12,490 $(57.1 \%)$ were STEMI patients and the remaining 9400 (42.9\%) were NSTEMI patients (Fig. 1). Any information concerning adverse events in these 21,890 participants with AMI including the time intervals and the types of events after the index PCI, which occurred during the follow-up period, was monitored at the outpatient clinic, by phone calls, or by reviewing the patients' charts at each participating center, and all participants completed a 2-year clinical follow-up [14].

\section{PCI procedure and medical treatment}

Diagnostic coronary angiography and PCI were performed after an administration of unfractionated heparin (50-100 IU/kg) according to standard technique [15]. Before PCI, all patients received loading doses of acetylsalicylic acid (ASA) 200-300 mg and clopidogrel 300-600 mg; alternatively, ticagrelor $180 \mathrm{mg}$ or prasugrel $60 \mathrm{mg}$ was administered. Moreover, dual antiplatelet therapy (DAPT), such as a daily dose of $100 \mathrm{mg}$ ASA and $75 \mathrm{mg}$ clopidogrel or ticagrelor $90 \mathrm{mg}$ twice daily or prasugrel 5-10 mg/day, was recommended for more than 12 months after PCI. The choice of triple antiplatelet therapy (cilostazol $100 \mathrm{mg}$ twice daily added to DAPT) was determined by the discretion of the individual operators [10]. The statins and their doses were as follows: $10-40 \mathrm{mg}$ of atorvastatin, $5-10 \mathrm{mg}$ of rosuvastatin, $2-4 \mathrm{mg}$ of pitavastatin, $10-40 \mathrm{mg}$ of simvastatin, $10-40 \mathrm{mg}$ of pravastatin, $80 \mathrm{mg}$ fluvastatin, and 50-100 mg lovastatin per day. The RASI used and their doses were as follows: $12.5-$ $-75 \mathrm{mg}$ of captopril, $2.5-10 \mathrm{mg}$ of ramipril, $2-8 \mathrm{mg}$ of perindopril, $1.25-5 \mathrm{mg}$ of cilazapril, $5-10 \mathrm{mg}$ of imidapril, $7.5-15 \mathrm{mg}$ of moexipril, $2.5-10 \mathrm{mg}$ of 


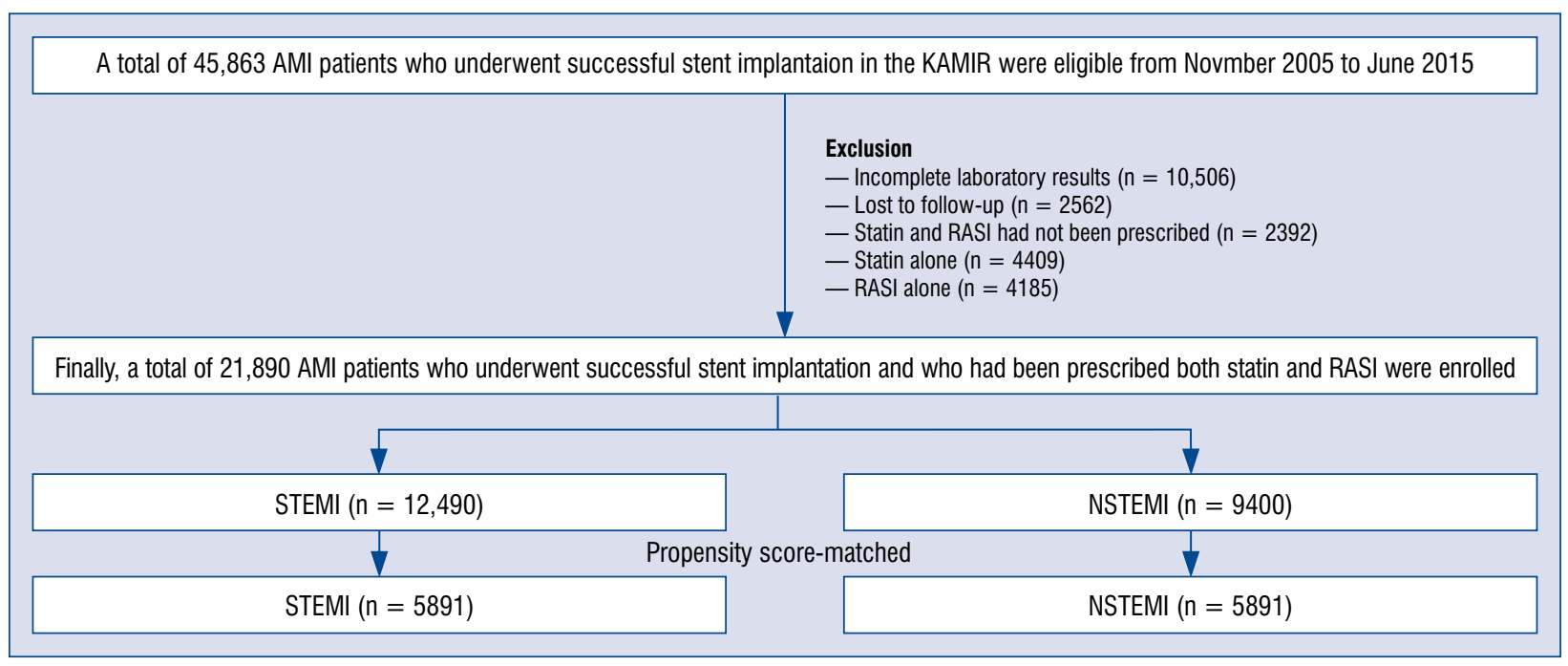

Figure 1. Flow chart; AMI - acute myocardial infarction; KAMIR - Korea Acute Myocardial Infarction Registry; NSTEMI — non-ST-segment elevation myocardial infarction; RASI — renin-angiotensin system inhibitor; STEMI — ST-segment elevation myocardial infarction.

enalapril, $5-10 \mathrm{mg}$ of lisinopril, $10 \mathrm{mg}$ of fosinopril, 3.75-7.5 mg of zofenopril, 25-100 mg of losartan, $150-300 \mathrm{mg}$ of irbesartan, 40-160 mg of valsartan, 40-80 mg of telmisartan, 10-20 mg of olmesartan, 4-32 mg of candesartan, $600 \mathrm{mg}$ of eprosartan, and 30-120 mg of fimasartan per day.

\section{Study definitions and clinical outcomes}

According to the current guidelines [6, 8], STEMI was defined as follows: ongoing chest pain and admission electrocardiogram showing ST-segment elevation in at least 2 contiguous leads of $\geq 2 \mathrm{~mm}(0.2 \mathrm{mV})$ in men or $\geq 1.5 \mathrm{~mm}(0.15 \mathrm{mV})$ in women in leads $\mathrm{V} 2-\mathrm{V} 3$ and/or of $\geq 1 \mathrm{~mm}$ $(0.1 \mathrm{mV})$ in other contiguous chest leads or the limb leads; or new-onset left bundle branch block [8]. NSTEMI was defined as follows: absence of persistent ST-segment elevation with increased cardiac biomarkers and appropriate clinical context [6]. In the present study, early invasive treatment strategy was defined as performing PCI within 24 hours after admission [10]. The major clinical endpoint was the occurrence of MACEs, defined as all-cause death, recurrent myocardial infarction (Re-MI), and any coronary repeat revascularization during a 2-year follow-up period. Any coronary repeat revascularization comprised target lesion revascularization (TLR), TVR, and non-TVR. Allcause death was classified as cardiac death (CD) or non-CD. Re-MI was defined as the presence of clinical symptoms, electrocardiographic changes, or abnormal imaging findings of MI combined with an increase in the creatine kinase myocardial band
(CK-MB) fraction above the upper normal limits or an increase in troponin-T/troponin-I to greater than the $99^{\text {th }}$ percentile of the upper normal limit after the index PCI [10]. The definitions of TLR, TVR, and non-TVR were previously published [10].

\section{Statistical analyses}

For continuous variables, differences between groups were evaluated with the unpaired t-test. Data are expressed as mean \pm standard deviation. For discrete variables, differences are expressed as counts and percentages, and were analyzed with $\chi^{2}$ test or Fisher's exact test between the groups. Various clinical outcomes were estimated using the Kaplan-Meier method, and differences between the two groups were compared by the log-rank test. To adjust for potential confounders, propensity scorematched (PSM) analysis was performed. We tested all available variables that could be of potential relevance: baseline clinical, laboratory, angiographic, and procedural characteristics (Table 1). The C-statistic for PSM was 0.821. Subjects were matched with a caliper width equal to 0.01 . The procedure yielded 5891 matched pairs except for the serum levels of CK-MB and troponin-I. Many patients were excluded during this PSM analysis; to overcome this limitation, we performed multivariate analysis. Any variable with a $\mathrm{p}$ value of $<0.001$ in univariate analysis and conventional risk factors of a poor outcome in the AMI population were considered as potential confounding factors and entered into the multivariate analysis (Table 2). Using Kaplan-Meier analysis, the differences 
Table 1. Baseline clinical, laboratory, angiographic, and procedural characteristics.

\begin{tabular}{|c|c|c|c|c|c|c|}
\hline \multirow[t]{2}{*}{ Variables } & \multicolumn{3}{|c|}{ Total study population } & \multicolumn{3}{|c|}{ Propensity score-matched patients } \\
\hline & $\begin{array}{c}\text { STEMI } \\
(\mathrm{n}=12,490)\end{array}$ & $\begin{array}{c}\text { NSTEMI } \\
(\mathrm{n}=9400)\end{array}$ & $\mathbf{P}$ & $\begin{array}{c}\text { STEMI } \\
(\mathrm{n}=\mathbf{5 8 9 1})\end{array}$ & $\begin{array}{c}\text { NSTEMI } \\
(\mathrm{n}=5891)\end{array}$ & $\mathbf{P}$ \\
\hline Age [years] & $61.9 \pm 12.6$ & $64.2 \pm 12.0$ & $<0.001$ & $63.3 \pm 12.5$ & $63.3 \pm 12.2$ & 0.654 \\
\hline Men & $9638(77.2 \%)$ & $6622(70.4 \%)$ & $<0.001$ & $4361(74.0 \%)$ & $4350(73.8 \%)$ & 0.817 \\
\hline LVEF [\%] & $51.5 \pm 10.8$ & $54.6 \pm 11.1$ & $<0.001$ & $53.5 \pm 10.9$ & $53.8 \pm 11.0$ & 0.136 \\
\hline BMI $\left[\mathrm{kg} / \mathrm{m}^{2}\right]$ & $24.2 \pm 3.1$ & $24.2 \pm 3.1$ & 0.448 & $24.1 \pm 3.0$ & $24.2 \pm 3.0$ & 0.525 \\
\hline $\mathrm{SBP}[\mathrm{mmHg}]$ & $129.1 \pm 27.8$ & $135.1 \pm 26.3$ & $<0.001$ & $132.6 \pm 26.8$ & $132.8 \pm 26.1$ & 0.587 \\
\hline $\mathrm{DBP}[\mathrm{mmHg}]$ & $79.2 \pm 16.8$ & $81.1 \pm 15.3$ & $<0.001$ & $80.2 \pm 15.8$ & $80.4 \pm 15.5$ & 0.624 \\
\hline Cardiogenic shock & $645(5.2 \%)$ & $154(1.6 \%)$ & $<0.001$ & $129(2.2 \%)$ & $137(2.3 \%)$ & 0.620 \\
\hline CPR on admission & $358(2.9 \%)$ & $133(1.4 \%)$ & $<0.001$ & $119(2.0 \%)$ & $105(1.8 \%)$ & 0.345 \\
\hline Hypertension & $5831(46.7 \%)$ & $5117(54.4 \%)$ & $<0.001$ & $3010(51.1 \%)$ & $3004(51.0 \%)$ & 0.912 \\
\hline Diabetes mellitus & $2944(23.6 \%)$ & $2762(29.4 \%)$ & $<0.001$ & $1568(26.6 \%)$ & $1582(26.9 \%)$ & 0.771 \\
\hline Dyslipidemia & $1383(11.1 \%)$ & $1246(13.3 \%)$ & $<0.001$ & $723(12.3 \%)$ & $692(11.7 \%)$ & 0.380 \\
\hline Previous MI & $334(2.7 \%)$ & $458(4.9 \%)$ & $<0.001$ & $226(3.8 \%)$ & $213(3.6 \%)$ & 0.527 \\
\hline Previous $\mathrm{PCl}$ & $514(4.1 \%)$ & $701(7.5 \%)$ & $<0.001$ & $332(5.6 \%)$ & $332(5.6 \%)$ & 1.000 \\
\hline Previous CABG & $38(0.3 \%)$ & $63(0.7 \%)$ & $<0.001$ & $27(0.5 \%)$ & $29(0.5 \%)$ & 0.789 \\
\hline Previous HF & $71(0.6 \%)$ & $131(1.4 \%)$ & $<0.001$ & $46(0.8 \%)$ & $41(0.7 \%)$ & 0.591 \\
\hline Previous CVA & $637(5.1 \%)$ & $670(7.1 \%)$ & $<0.001$ & $353(6.0 \%)$ & $362(6.1 \%)$ & 0.728 \\
\hline Current smokers & $6067(48.6 \%)$ & $3620(38.5 \%)$ & $<0.001$ & $2549(43.3 \%)$ & $2546(43.2 \%)$ & 0.956 \\
\hline CK-MB [mg/dL] & $171.3 \pm 216.0$ & $65.3 \pm 168.6$ & $<0.001$ & $101.4 \pm 214.8$ & $82.0 \pm 207.2$ & $<0.001$ \\
\hline Troponin-I [ng/mL] & $59.6 \pm 126.9$ & $23.1 \pm 43.5$ & $<0.001$ & $37.8 \pm 155.3$ & $28.6 \pm 52.4$ & $<0.001$ \\
\hline NT-proBNP [pg/mL] & $1497.5 \pm 2832.4$ & $2101.7 \pm 4751.4$ & $<0.001$ & $1748.2 \pm 3636.7$ & $1761.3 \pm 3162.3$ & 0.836 \\
\hline hs-CRP [mg/dL] & $10.9 \pm 51.0$ & $11.9 \pm 55.9$ & 0.207 & $11.5 \pm 52.0$ & $11.1 \pm 45.3$ & 0.616 \\
\hline Serum creatinine $[\mathrm{mg} / \mathrm{L}]$ & $1.05 \pm 1.00$ & $1.09 \pm 1.16$ & 0.007 & $1.06 \pm 1.00$ & $1.07 \pm 1.07$ & 0.667 \\
\hline Blood glucose [mg/dL] & $170.8 \pm 72.3$ & $158.5 \pm 76.2$ & $<0.001$ & $162.4 \pm 65.1$ & $161.2 \pm 79.5$ & 0.348 \\
\hline Total cholesterol [mg/dL] & $186.8 \pm 43.4$ & $185.0 \pm 45.6$ & 0.004 & $185.9 \pm 43.6$ & $185.7 \pm 45.9$ & 0.818 \\
\hline Triglyceride [mg/L] & $136.4 \pm 113.3$ & $136.3 \pm 108.3$ & 0.943 & $136.4 \pm 112.8$ & $137.2 \pm 113.6$ & 0.702 \\
\hline HDL cholesterol [mg/L] & $44.2 \pm 19.3$ & $43.5 \pm 15.4$ & 0.001 & $43.8 \pm 19.4$ & $43.6 \pm 16.3$ & 0.638 \\
\hline LDL cholesterol [mg/L] & $119.0 \pm 38.8$ & $117.7 \pm 39.4$ & 0.016 & $117.9 \pm 38.8$ & $117.8 \pm 39.9$ & 0.874 \\
\hline \multicolumn{7}{|l|}{ Discharge medications: } \\
\hline ASA & $12430(99.5 \%)$ & $9341(99.4 \%)$ & 0.142 & $5859(99.5 \%)$ & $5856(99.4 \%)$ & 0.713 \\
\hline Clopidogrel & $11212(89.8 \%)$ & $8340(88.7 \%)$ & 0.013 & $5263(89.3 \%)$ & $5247(89.1 \%)$ & 0.635 \\
\hline Ticagrelor & $727(5.8 \%)$ & $625(6.6 \%)$ & 0.012 & $366(6.2 \%)$ & $365(6.2 \%)$ & 0.970 \\
\hline Prasugrel & $438(3.5 \%)$ & $344(3.7 \%)$ & 0.547 & $208(3.5 \%)$ & $223(3.8 \%)$ & 0.462 \\
\hline Cilostazol & $3077(24.6 \%)$ & $2202(23.4 \%)$ & 0.038 & $1366(23.2 \%)$ & $1386(23.5 \%)$ & 0.663 \\
\hline Beta-blockers & $10824(86.7 \%)$ & $8082(86.0 \%)$ & 0.145 & $5094(86.5 \%)$ & $5114(86.8 \%)$ & 0.588 \\
\hline CCB & $549(4.4 \%)$ & $824(8.8 \%)$ & $<0.001$ & $354(6.0 \%)$ & $350(5.9 \%)$ & 0.876 \\
\hline \multicolumn{7}{|c|}{ Angiographic and procedural characteristics } \\
\hline $\mathrm{PCl}$ within 24 hours & $11668(93.4)$ & $7444(79.2)$ & $<0.001$ & $5203(88.3)$ & $5213(88.5)$ & 0.774 \\
\hline \multicolumn{7}{|l|}{ Infarct-related artery: } \\
\hline Left main & $112(0.9 \%)$ & $188(2.0 \%)$ & $<0.001$ & $73(1.2 \%)$ & $75(1.3 \%)$ & 0.869 \\
\hline Left anterior descending & $6535(52.3 \%)$ & $3988(42.4 \%)$ & $<0.001$ & $2885(49.0 \%)$ & $2869(48.7 \%)$ & 0.768 \\
\hline Left circumflex & $1138(9.1 \%)$ & $2603(27.7 \%)$ & $<0.001$ & $932(15.8 \%)$ & $932(15.8 \%)$ & 1.000 \\
\hline Right coronary artery & $4705(37.7 \%)$ & $2621(27.9 \%)$ & $<0.001$ & $2001(34.0 \%)$ & $2015(34.2 \%)$ & 0.786 \\
\hline \multicolumn{7}{|l|}{ Treated vessel: } \\
\hline Left main & $196(1.6 \%)$ & $332(3.5 \%)$ & $<0.001$ & $133(2.3 \%)$ & $129(2.2 \%)$ & 0.803 \\
\hline Left anterior descending & $7414(59.4 \%)$ & $5202(55.3 \%)$ & $<0.001$ & $3436(58.3 \%)$ & $3444(58.5 \%)$ & 0.881 \\
\hline Left circumflex & $2027(16.2 \%)$ & $3733(39.7 \%)$ & $<0.001$ & $1535(26.1 \%)$ & $1504(25.5 \%)$ & 0.514 \\
\hline Right coronary artery & $5301(42.4 \%)$ & $3486(37.1 \%)$ & $<0.001$ & $2415(41.0 \%)$ & $2414(41.0 \%)$ & 0.985 \\
\hline
\end{tabular}


Table 1 (cont.). Baseline clinical, laboratory, angiographic, and procedural characteristics.

\begin{tabular}{|c|c|c|c|c|c|c|}
\hline \multirow[t]{2}{*}{ Variables } & \multicolumn{3}{|c|}{ Total study population } & \multicolumn{3}{|c|}{ Propensity score-matched patients } \\
\hline & $\begin{array}{c}\text { STEMI } \\
(\mathrm{n}=12,490)\end{array}$ & $\begin{array}{c}\text { NSTEMI } \\
(\mathrm{n}=9400)\end{array}$ & $\mathbf{P}$ & $\begin{array}{c}\text { STEMI } \\
(\mathrm{n}=\mathbf{5 8 9 1})\end{array}$ & $\begin{array}{c}\text { NSTEMI } \\
(\mathrm{n}=\mathbf{5 8 9 1})\end{array}$ & $\mathbf{P}$ \\
\hline \multicolumn{7}{|l|}{ Treated vessel: } \\
\hline Left main & $196(1.6 \%)$ & $332(3.5 \%)$ & $<0.001$ & $133(2.3 \%)$ & $129(2.2 \%)$ & 0.803 \\
\hline Left anterior descending & $7414(59.4 \%)$ & $5202(55.3 \%)$ & $<0.001$ & $3436(58.3 \%)$ & $3444(58.5 \%)$ & 0.881 \\
\hline Left circumflex & $2027(16.2 \%)$ & $3733(39.7 \%)$ & $<0.001$ & $1535(26.1 \%)$ & $1504(25.5 \%)$ & 0.514 \\
\hline Right coronary artery & $5301(42.4 \%)$ & $3486(37.1 \%)$ & $<0.001$ & $2415(41.0 \%)$ & $2414(41.0 \%)$ & 0.985 \\
\hline \multicolumn{7}{|l|}{ ACC/AHA lesion type: } \\
\hline Type B1 & $1745(14.0 \%)$ & $1448(15.4 \%)$ & 0.003 & $875(14.9 \%)$ & $908(15.4 \%)$ & 0.396 \\
\hline Type B2 & $3706(29.7 \%)$ & $3418(36.4 \%)$ & $<0.001$ & $1969(33.4 \%)$ & $1984(33.7 \%)$ & 0.770 \\
\hline Type C & $5911(47.3 \%)$ & $3752(39.9 \%)$ & $<0.001$ & $2543(43.2 \%)$ & $2488(42.2 \%)$ & 0.306 \\
\hline \multicolumn{7}{|c|}{ Extent of coronary artery disease: } \\
\hline 1-vessel & $6534(52.3 \%)$ & $4040(43.2 \%)$ & $<0.001$ & $2809(47.7 \%)$ & $2774(47.1 \%)$ & 0.518 \\
\hline 2-vessel & $3701(29.6 \%)$ & $3212(34.2 \%)$ & $<0.001$ & $1882(31.9 \%)$ & $1895(32.2 \%)$ & 0.797 \\
\hline$\geq 3$-vessel & $2255(18.1 \%)$ & $2148(22.9 \%)$ & $<0.001$ & $1200(20.4 \%)$ & $1222(20.7 \%)$ & 0.616 \\
\hline Multi-vessel disease & $5956(47.7 \%)$ & $5360(57.0 \%)$ & $<0.001$ & $3082(52.3 \%)$ & $3117(52.9 \%)$ & 0.518 \\
\hline \multicolumn{7}{|l|}{ Drug-eluting stents: } \\
\hline BMS & $834(6.7 \%)$ & $534(5.7 \%)$ & 0.003 & $344(5.8 \%)$ & $358(6.1 \%)$ & 0.586 \\
\hline SES & $1941(15.5 \%)$ & $1207(12.8 \%)$ & $<0.001$ & $782(13.3 \%)$ & $815(13.8 \%)$ & 0.374 \\
\hline PES & $1667(13.3 \%)$ & $1123(11.9 \%)$ & 0.002 & $747(12.7 \%)$ & $716(12.2 \%)$ & 0.386 \\
\hline ZES & $2780(22.3 \%)$ & $2006(21.3 \%)$ & 0.104 & $1254(21.3 \%)$ & $1273(21.6 \%)$ & 0.670 \\
\hline EES & $3548(28.4 \%)$ & $3168(33.7 \%)$ & $<0.001$ & $1939(32.9 \%)$ & $1885(32.0 \%)$ & 0.288 \\
\hline BES & $1012(8.1 \%)$ & $1002(10.7 \%)$ & $<0.001$ & $543(9.2 \%)$ & $541(9.2 \%)$ & 0.949 \\
\hline Stent diameter [mm] & $3.20 \pm 0.42$ & $3.10 \pm 0.42$ & $<0.001$ & $3.14 \pm 0.41$ & $3.14 \pm 0.42$ & 0.572 \\
\hline Stent length [mm] & $26.1 \pm 9.1$ & $26.7 \pm 11.2$ & $<0.001$ & $26.5 \pm 9.9$ & $26.5 \pm 10.4$ & 0.965 \\
\hline Number of stents & $1.41 \pm 0.72$ & $1.60 \pm 0.88$ & $<0.001$ & $1.51 \pm 0.80$ & $1.52 \pm 0.79$ & 0.811 \\
\hline
\end{tabular}

Values are mean \pm standard deviation or number (\%). The p values for continuous data were obtained from analysis of the unpaired t-test. The $\mathrm{p}$ values for categorical data were obtained from chi-square test. STEMI — ST-segment elevation myocardial infarction; NSTEMI - non-STEMI; LVEF — left ventricular ejection fraction; BMI — body mass index; SBP — systolic blood pressure; DBP — diastolic blood pressure; $\mathrm{CPR}$ - cardiopulmonary resuscitation; $\mathrm{PCl}$ - percutaneous coronary intervention; $\mathrm{MI}$ - myocardial infarction; CABG — coronary artery bypass graft; CVA — cerebrovascular accident; HF — heart failure; CK-MB - creatine kinase myocardial band; NT-proBNP - N-terminal pro-B-type natriuretic peptide; hs-CRP — high-sensitivity-C-reactive protein; HDL — high-density lipoprotein; LDL — low-density lipoprotein; ASA - acetylsalicylic acid; CCB - calcium channel blockers; ACC/AHA - American College of Cardiology/American Heart Association; BMS - bare-metal stent; SES - sirolimus-eluting stent; PES - paclitaxel-eluting stent; ZES - zotarolimus-eluting stent; EES - everolimus-eluting stent; BES - biolimus-eluting stent

between the groups were compared using the logrank test. For all analyses, a two-sided $\mathrm{p}<0.05$ was considered statistically significant. All statistical analyses were performed using SPSS software, version 20 (IBM; Armonk, NY, USA) [7].

\section{Results}

Baseline clinical, laboratory, angiographic, and procedural characteristics

In the total study population, the mean age of the NSTEMI group was greater than that of the STEMI group $(64.2 \pm 12.0$ years vs. $61.9 \pm$ 12.6 years, $p<0.001$, Table 1 ). The following val- ues were higher in the STEMI group than in the NSTEMI group: number of men; value of cardiogenic shock, cardiopulmonary resuscitation (CPR), and current smokers; levels of CK-MB, troponin I, blood glucose, total cholesterol, high-density lipoprotein (HDL)-cholesterol, and low-density lipoprotein (LDL)-cholesterol; prescription rates of clopidogrel and cilostazol; numbers of PCI within 24 hours, left anterior descending artery (LAD, infract-related artery [IRA] and treated vessel) and right coronary artery (RCA, IRA and treated vessel); and numbers of American College of Cardiology/American Heart Association (ACC/AHA) type $\mathrm{C}$ and 1-vessel disease. By contrast, the NSTEMI 
Table 2. Clinical outcomes by Kaplan-Meier analysis and Cox-proportional hazard ratio analysis up to 2 years.

\begin{tabular}{|c|c|c|c|c|c|}
\hline \multirow[t]{2}{*}{ Outcomes } & \multicolumn{5}{|c|}{ Cumulative events at 2-year (\%) } \\
\hline & STEMI & NSTEMI & Log-rank & Hazard ratio $(95 \% \mathrm{Cl})$ & $\mathbf{P}$ \\
\hline \multicolumn{6}{|l|}{ Total study population } \\
\hline MACEs & $851(7.2)$ & $728(8.3)$ & 0.003 & $1.159(1.050-1.280)$ & 0.003 \\
\hline All-cause death & $228(1.9)$ & $255(2.9)$ & $<0.001$ & $1.512(1.265-1.808)$ & $<0.001$ \\
\hline Cardiac death & $164(1.4)$ & $170(1.9)$ & 0.002 & $1.398(1.128-1.733)$ & 0.002 \\
\hline Re-MI & $181(1.5)$ & $149(1.7)$ & 0.319 & $1.117(0.899-1.387)$ & 0.319 \\
\hline Total repeat revascularization: & $507(4.3)$ & $399(4.7)$ & 0.310 & $1.070(0.939-1.220)$ & 0.310 \\
\hline TLR & $160(1.4)$ & $122(1.4)$ & 0.774 & $1.035(0.818-1.310)$ & 0.774 \\
\hline TVR & $294(2.5)$ & $244(2.8)$ & 0.153 & $1.132(0.955-1.341)$ & 0.154 \\
\hline Non-TVR & $222(1.9)$ & $162(1.9)$ & 0.933 & $0.991(0.810-1.214)$ & 0.933 \\
\hline \multicolumn{6}{|l|}{ Propensity score-matched patients } \\
\hline MACEs & $414(7.4)$ & $452(8.2)$ & 0.132 & $1.108(0.969-1.266)$ & 0.132 \\
\hline All-cause death & $114(2.0)$ & $158(2.9)$ & 0.005 & $1.407(1.106-1.790)$ & 0.005 \\
\hline Cardiac death & $82(1.4)$ & $106(1.9)$ & 0.046 & $1.311(0.983-1.749)$ & 0.046 \\
\hline Re-MI & $94(1.7)$ & $90(1.7)$ & 0.847 & $0.972(0.728-1.298)$ & 0.847 \\
\hline Total repeat revascularization: & $241(4.4)$ & $252(4.7)$ & 0.485 & $1.065(0.893-1.271)$ & 0.485 \\
\hline TLR & $85(1.5)$ & $71(1.3)$ & 0.308 & $0.849(0.619-1.163)$ & 0.308 \\
\hline TVR & $150(2.7)$ & $150(2.8)$ & 0.871 & $1.019(0.813-1.278)$ & 0.871 \\
\hline Non-TVR & $98(1.8)$ & $107(2.0)$ & 0.444 & $1.113(0.846-1.464)$ & 0.444 \\
\hline \multicolumn{6}{|l|}{ Multivariate analysis* } \\
\hline MACEs & $851(7.2)$ & $728(8.3)$ & 0.003 & $1.081(0.965-1.210)$ & 0.178 \\
\hline All-cause death & $228(1.9)$ & $255(2.9)$ & $<0.001$ & $1.528(1.264-1.852)$ & 0.001 \\
\hline Cardiac death & $164(1.4)$ & $170(1.9)$ & 0.002 & $1.406(1.146-1.802)$ & 0.020 \\
\hline Re-MI & $181(1.5)$ & $149(1.7)$ & 0.319 & $1.021(0.798-1.308)$ & 0.866 \\
\hline Total repeat revascularization: & $507(4.3)$ & $399(4.7)$ & 0.310 & $0.975(0.839-1.134)$ & 0.746 \\
\hline TLR & $160(1.4)$ & $122(1.4)$ & 0.774 & $0.857(0.653-1.124)$ & 0.264 \\
\hline TVR & $294(2.5)$ & $244(2.8)$ & 0.153 & $0.979(0.805-1.190)$ & 0.830 \\
\hline Non-TVR & $222(1.9)$ & $162(1.9)$ & 0.933 & $0.963(0.764-1.213)$ & 0.748 \\
\hline
\end{tabular}

*Adjusted by age, men, LVEF, SBP, DBP, cardiogenic shock, CPR on admission, hypertension, diabetes, dyslipidemia, previous history of MI, $\mathrm{PCl}, \mathrm{CABG}, \mathrm{HF}$, and CVA, current smoker, serum level of CK-MB, troponin I, NT-proBNP, blood glucose, CCB, PCI within 24 hours, IRA, treated vessel, ACC/AHA type B2, and C lesion, the extent of coronary artery disease, types of stents (SES, EES, and BES), stent diameter, stent length, and number of stents. STEMI — ST-segment elevation myocardial infarction; NSTEMI — non-STEMI; CI — confidence interval;

MACE - major adverse cardiac events; Re-MI - re-myocardial infarction; TLR - target lesion revascularization: TVR - target vessel revascularization; Non-TVR - non-TVR; LVEF — left ventricular ejection fraction; SBP — systolic blood pressure; DBP — diastolic blood pressure; $\mathrm{CPR}$ - cardiopulmonary resuscitation; $\mathrm{MI}$ - myocardial infarction; $\mathrm{PCl}$ - percutaneous coronary intervention; CABG - coronary artery bypass graft; HF — heart failure; CVA - cerebrovascular accidents; CK-MB — creatine kinase myocardial band; NT-proBNP - N-terminal pro-B-type natriuretic peptide; CCB - calcium channel blockers; IRA — infarct-related artery; ACC/AHA - American College of Cardiology/ /American Heart Association; SES — sirolimus-eluting stent; EES — everolimus-eluting stents; BES — biolimus-eluting stents

group showed higher values than the STEMI group for the following: left ventricular ejection fraction (LVEF, $54.6 \pm 11.1 \%$ vs. $51.5 \pm 10.8 \%$, $\mathrm{p}<0.001$ ); systolic blood pressure; diastolic blood pressure; number of patients with hypertension, diabetes, dyslipidemia, previous history of MI, PCI, coronary artery bypass graft, heart failure (HF), and cerebrovascular accident; levels of serum $\mathrm{N}$-terminal pro-B-type natriuretic peptide and se- rum creatinine; prescription rates of ticagrelor and calcium channel blockers; the number of left main coronary artery (LM, IRA and treated vessel), left circumflex artery (LCx, IRA and treated vessel); and the frequency of multi-vessel disease (MVD). Bare-metal stents (BMS) and first-generation DESs were more frequently deployed in the STEMI group, and the everolimus-eluting stents and biolimus-eluting stents were more frequently de- 


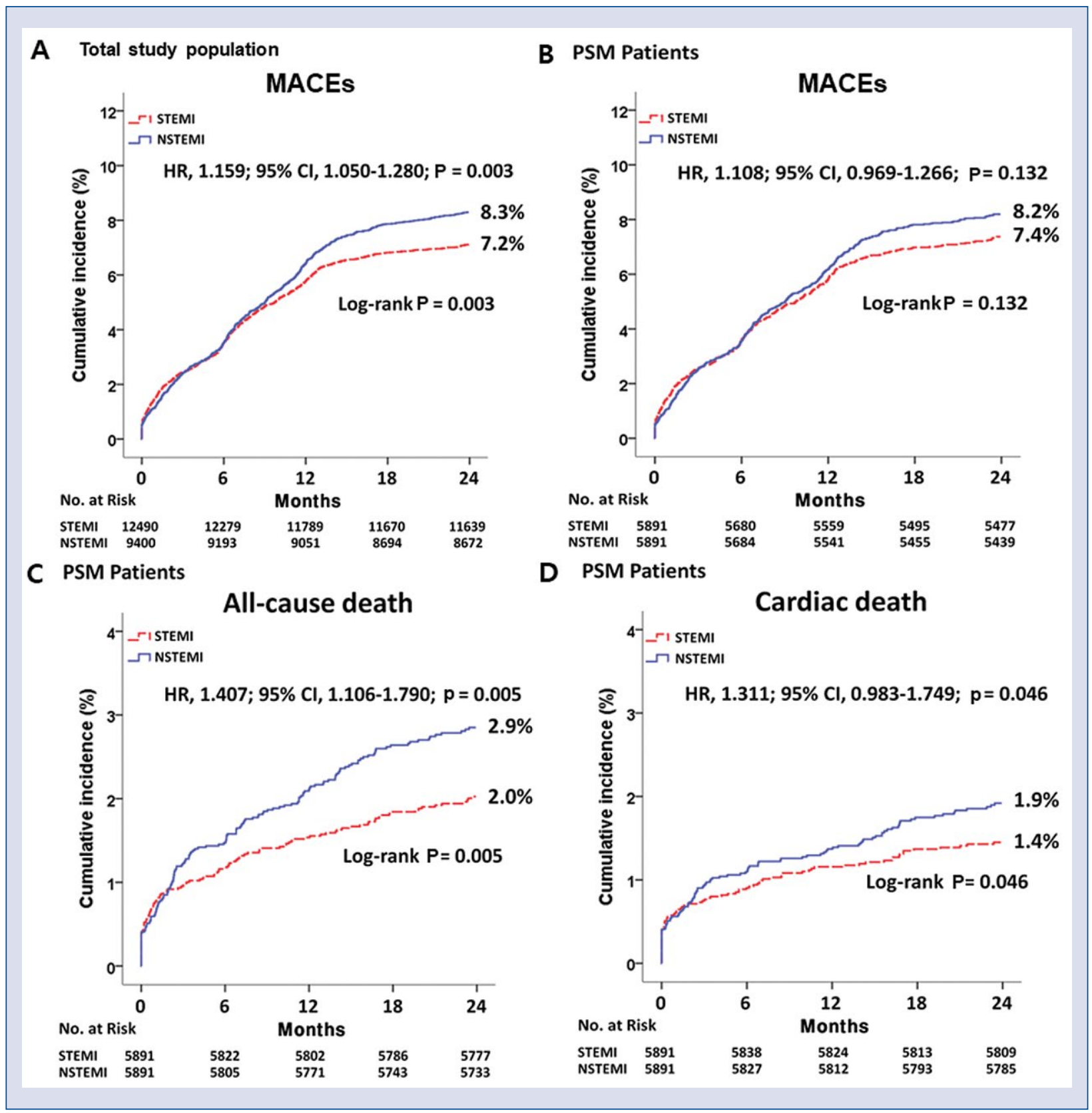

Figure 2. Kaplan-Meier analysis for major adverse cardiac events (MACEs) (A, B), all-cause death (C), and cardiac death (D); PSM - propensity score-matched; HR - hazard ratio; Cl - confidence interval; NSTEMI - non-ST-segment elevation myocardial infarction; STEMI - ST-segment elevation myocardial infarction.

ployed in the NSTEMI group. After PSM analysis, baseline differences between the two groups were well balanced. However, the blood levels of CK-MB and troponin I levels were not well balanced.

\section{Clinical outcomes}

In the total study population, the cumulative incidence of MACEs (hazard ratio [HR] 1.159; 95\% confidence interval [CI] 1.050-1.280; $\mathrm{p}=0.003$, Fig. 2A), all-cause death (HR 1.512;
95\% CI 1.265-1.808; p < 0.001), and CD (HR $1.398 ; 95 \%$ CI $1.128-1.733 ; \mathrm{p}=0.002$ ) were higher in the NSTEMI group than in the STEMI group. After PSM analysis, the cumulative incidence of MACEs was not significantly different between the groups (Fig. 2B). However, the cumulative incidences of all-cause death (HR 1.407; 95\% CI 1.106-1.790; $\mathrm{p}=0.005$, Fig. $2 \mathrm{C}$ ) and $\mathrm{CD}$ (HR $1.311 ; 95 \%$ CI $0.983-1.749 ; \mathrm{p}=0.046$, Fig. $2 \mathrm{D}$ ) were significantly higher in the NSTEMI group 
than in the STEMI group. Before and after PSM analysis, the cumulative incidences of Re-MI, any repeat revascularization, TLR, TVR, and non-TVR were not statistically different between the groups. Figure 3 shows subgroup analysis for MACEs at 2 years. In cases of male sex (HR 1.13; 95\% CI $1.00-1.27 ; \mathrm{p}=0.047)$, low LVEF $(<50 \%$, HR 1.47; 95\% CI 1.26-1.71; $\mathrm{p}<0.001)$, cardiogenic shock (HR 1.17; 95\% CI 1.06-1.29; $\mathrm{p}=0.002$ ), and PCI within 24 hours (HR 1.15; 95\% CI 1.03-1.28; $\mathrm{p}=0.012$ ) statins combined with RASI showed greater reduction in MACEs for patients with STEMI than for those with NSTEMI. Advanced age ( $\geq 65$ years), low LVEF $(<50 \%)$, diabetes, CPR on admission, N-terminal pro-B-type natriuretic peptide, serum creatinine, total cholesterol, triglyceride, LDL-cholesterol, PCI within 24 hours, $\mathrm{BMS}$, and MVD were meaningful independent risk factors for both all-cause death and CD in PSM patients (Table 3 ).

\section{Discussion}

According to current guidelines [5, 6, 8, 9], more than $80 \%$ of the patients with AMI received statin therapy in Korea [16]. Similarly, more than $50 \%$ of these patients received RASI therapy to reduce cardiovascular mortality [17]. However, the comparative studies regarding long-term clinical outcomes of statin with RASI combination therapy between STEMI and NSTEMI after stent implantation have not been reported. We believe this may be the first report focusing on this issue. Moreover, the present study confirms that statin combined with RASI was more effective in patients with STEMI rather than in patients with NSTEMI with respect to all-cause death and $\mathrm{CD}$ rates over a 2-year follow-up.

The key findings of this study are as follows: First, after PSM analysis, the cumulative incidences of all-cause death and CD were significantly higher in patients with NSTEMI than in those with STEMI after combined statin and RASI therapy. Second, the cumulative incidences of MACEs, Re-MI, and any repeat revascularization including TLR, TVR, and non-TVR were not significantly different between the two groups after PSM analysis. Third, advanced age ( $\geq 65$ years), male sex, low LVEF $(<50 \%)$, diabetes, CPR on admission, PCI within 24 hours, BMS, and MVD were independent risk factors for both all-cause death and $\mathrm{CD}$ in PSM patients.

Statins both reduce LDL-cholesterol and decrease the occurrences of cardiovascular death, non-fatal MI, and repeat coronary revascularization by inhibiting 3-hydroxy-3-methylglutarylcoenzyme A (HMG-CoA) reductase activity, as well as other not fully understood processes [3, $18,19]$. The beneficial effects of statins are evident in both STEMI and NSTEMI patients [5, 6, 8, 9]. However, data comparing the long-term prognosis of STEMI and NSTEMI patients, especially those focused on the usage of statins, are limited. In the era of DES, Kim et al. [7] demonstrated that MACEs and mortality reduction capacity of statin therapy was prominent compared with statin nonuse, and statins were more effective in patients with STEMI compared with NSTEMI. Regardless of STEMI or NSTEMI, RASI provides mortality reduction benefit by diminishing the rate of progressive LV dilation and remodeling, especially in patients with LV dysfunction [5, 6]. Even though Kim et al. [10] reported greater reduction in mortality following RASI in STEMI patients than in NSTEMI patients after successful PCI, long-term clinical outcome data comparing the status of STEMI and NSTEMI patients post successful stent implantation after combined statin and RASI therapy are also limited. Koh et al. [20] suggested that combined statins and RASI may improve endothelial function, insulin resistance, and atherosclerosis. In our study, the cumulative incidences of all-cause death (HR 1.407; 95\% CI 1.106-1.790; $\mathrm{p}=0.005)$ and CD (HR 1.311; 95\% CI 0.983-1.749; $\mathrm{p}=0.046)$ were also significantly higher in the NSTEMI group than in the STEMI group. Taken together, these results suggest that RASI monotherapy or statins combined with RASI was more beneficial for STEMI patients than for NSTEMI patients in terms of reduced mortality. Statins decrease the production of oxygen-derived free radicals by reducing LDL-cholesterol, increasing nitric oxide (NO) synthesis, promoting antioxidant effects, and inhibiting upregulation of angiotensin II type 1 (AT1) receptor expression. RASI inhibits binding of angiotensin II to the AT1 receptor and induces decreased production of oxygen-derived free radicals. Accumulated bradykinins after ACEI treatment lead to increased stimulation of NO production [20]. NO production may be a shared process for both statins and RASI. Kim et al. [10] showed that RASI after PSM was more effective in reducing all-cause death (HR 1.386; 95\% CI $1.114-1.725 ; \mathrm{p}=0.003)$ and $\mathrm{CD}$ (HR $1.358 ; 95 \% \mathrm{CI}$ $1.041-1.7770 ; \mathrm{p}=0.024)$ in patients with STEMI compared with NSTEMI after PSM. However, we found that statins combined with RASI did not show greater relative risk reduction of all-cause 
Table 3. Multivariate Cox-proportional regression analysis for predictors of all-cause death and cardiac death in propensity score-matched patients.

\begin{tabular}{|c|c|c|c|c|}
\hline \multirow[t]{2}{*}{ Variables } & \multicolumn{2}{|c|}{ All-cause death } & \multicolumn{2}{|c|}{ Cardiac death } \\
\hline & $\mathrm{HR}(95 \% \mathrm{CI})$ & $\mathbf{P}$ & HR $(95 \% \mathrm{CI})$ & $\mathbf{P}$ \\
\hline STEMI vs. NSTEMI & $2.822(2.102-3.789)$ & $<0.001$ & 2.643 (1.858-3.759) & $<0.001$ \\
\hline Age $\geq 65$ years & $2.617(1.945-3.521)$ & $<0.001$ & $2.491(1.748-3.551)$ & $<0.001$ \\
\hline Male & $1.009(0.774-1.315)$ & 0.949 & $1.057(0.770-1.450)$ & 0.733 \\
\hline LVEF $<50 \%$ & $1.961(1.531-2.510)$ & $<0.001$ & $1.887(1.403-2.540)$ & $<0.001$ \\
\hline Systolic blood pressure & $0.991(0.982-0.999)$ & 0.024 & $0.992(0.983-1.002)$ & 0.121 \\
\hline Diastolic blood pressure & $1.012(0.999-1.026)$ & 0.072 & $1.010(0.994-1.027)$ & 0.204 \\
\hline Hypertension & $1.186(0.916-1.535)$ & 0.196 & $1.236(0.905-1.689)$ & 0.183 \\
\hline Diabetes mellitus & $1.522(1.184-1.956)$ & 0.001 & $1.453(1.074-1.967)$ & 0.015 \\
\hline Dyslipidemia & $1.140(0.758-1.715)$ & 0.528 & $1.086(0.693-1.702)$ & 0.720 \\
\hline Cardiogenic shock & $1.074(0.517-2.233)$ & 0.847 & $1.372(0.524-3.591)$ & 0.519 \\
\hline CPR on admission & $3.289(2.034-5.318)$ & $<0.001$ & $4.001(2.322-6.895)$ & $<0.001$ \\
\hline CK-MB & $1.000(0.999-1.001)$ & 0.814 & $1.000(0.999-1.001)$ & 0.969 \\
\hline Troponin-I & $1.000(1.000-1.001)$ & 0.407 & $1.000(1.000-1.001)$ & 0.497 \\
\hline NT-proBNP & $1.001(1.000-1.002)$ & $<0.001$ & 1.002 (1.001-1.003) & $<0.001$ \\
\hline hs-CRP & $1.001(0.999-1.003)$ & 0.256 & $1.000(0.998-1.003)$ & 0.766 \\
\hline Serum creatinine & $1.128(1.074-1.186)$ & $<0.001$ & 1.124 (1.057-1.195) & $<0.001$ \\
\hline Total cholesterol & $0.994(0.991-0.997)$ & $<0.001$ & $0.992(0.989-0.996)$ & $<0.001$ \\
\hline Triglyceride & $0.996(0.994-0.998)$ & $<0.001$ & $0.996(0.994-0.999)$ & 0.001 \\
\hline HDL-cholesterol & $0.993(0.982-1.003)$ & 0.173 & $0.994(0.981-1.006)$ & 0.308 \\
\hline LDL-cholesterol & $0.994(0.991-0.998)$ & 0.001 & $0.993(0.989-0.997)$ & 0.001 \\
\hline Beta-blocker & 1.562 (1.038-2.353) & 0.033 & $1.600(0.978-2.617)$ & 0.061 \\
\hline $\mathrm{PCl}$ within 24 hours & 1.483 (1.167-1.885) & 0.001 & 1.395 (1.046-1.860) & 0.024 \\
\hline LAD (IRA) & $1.122(0.752-1.676)$ & 0.572 & $1.020(0.633-1.643)$ & 0.934 \\
\hline LAD (treated) & $1.120(0.744-1.686)$ & 0.586 & $1.065(0.659-1.721)$ & 0.796 \\
\hline ACC/AHA type B2/C lesion & $1.124(0.824-1.533)$ & 0.461 & $1.007(0.703-1.442)$ & 0.970 \\
\hline BMS & $3.104(1.905-5.056)$ & $<0.001$ & $2.481(1.360-4.527)$ & 0.003 \\
\hline SES & $1.940(1.048-3.591)$ & 0.035 & $2.041(0.974-4.275)$ & 0.059 \\
\hline PES & $1.343(0.755-2.389)$ & 0.316 & $1.142(0.591-2.210)$ & 0.692 \\
\hline ZES & $1.128(0.702-1.813)$ & 0.618 & $1.084(0.616-1.909)$ & 0.780 \\
\hline EES & $1.150(0.725-1.824)$ & 0.552 & $1.112(0.642-1.924)$ & 0.706 \\
\hline BES & $1.066(0.606-1.874)$ & 0.825 & $1.196(0.598-2.390)$ & 0.613 \\
\hline MVD & $1.301(1.003-1.687)$ & 0.048 & $1.343(0.981-1.840)$ & 0.042 \\
\hline Stent diameter & $0.856(0.630-1.162)$ & 0.317 & $0.714(0.490-1.040)$ & 0.079 \\
\hline Stent length & $0.999(0.987-1.012)$ & 0.883 & $0.998(0.983-1.013)$ & 0.791 \\
\hline
\end{tabular}

HR - hazard ratio; $\mathrm{Cl}$ - confidence interval; STEMI — ST-segment elevation myocardial infarction; NSTEMI — non-STEMI; LVEF — left ventricular ejection fraction; CPR — cardiopulmonary resuscitation; CK-MB — creatine kinase myocardial band; NT-proBNP — N-terminal pro-B-type natriuretic peptide; hs-CRP — high sensitivity-C-reactive protein; HDL — high-density lipoprotein; LDL — low-density lipoprotein; $\mathrm{PCl}$ - percutaneous coronary intervention; LAD - left anterior descending coronary artery; IRA - infarct-related artery; ACC/AHA - American College of Cardiology/American Heart Association; BMS - bare-metal stent; SES - sirolimus-eluting stent; PES - paclitaxel-eluting stent; ZES - zotarolimus-eluting stent; EES - everolimus-eluting stent; BES — biolimus-eluting stent; MVD - multivessel disease

death $(40.7 \%$ vs. $38.6 \%)$ or CD $(31.1 \%$ vs. $35.8 \%)$ compared with the results of Kim et al. [10]. We speculated that one of the major causative factors for the similar relative risk reduction potency for all-cause death and CD in these two studies (Kim et al. [10] and the present study) is related, at least in part, with this shared NO production process [19]. This has included suggestion that this similar NO mediated protective mechanism may be attenuated their effects on endothelium each 
other. However, this supposition supports the need for further study to confirm these findings. Other possible factor for these results was the presence of BMS. BMS was not included in the Kim et al. [10] study. However, the proportion of BMS in the total study population was low, and BMS was an independent significant predictor for both all-cause death and cardiac death in PSM patients (Table 3). Nevertheless, with regard to the beneficial effect of statin monotherapy by reducing MACEs, all-cause death, and TVR in patients with STEMI [7], statin and RASI combination therapy showed an additional beneficial effect on reducing the cumulative incidence of CD (HR 1.311; 95\% CI $0.983-1.749 ; \mathrm{p}=0.046$ ) in this study.

On PSM analysis, many patients $(10,108 /$ $/ 21,890,46.2 \%)$ were excluded and the serum CK-MB and troponin-I levels were not wellmatched. To overcome these limitations, we performed standard multivariate analysis. Nevertheless, the results of multivariate analysis were similar to the results of the PSM analysis. After multivariate analysis, the cumulative incidences of all-cause death (HR 1.528; 95\% CI 1.264-1.852; $\mathrm{p}=0.001)$ and CD (HR 1.406; 95\% CI 1.146$-1.802 ; \mathrm{p}=0.020$ ) were significantly higher in NSTEMI patients than in STEMI patients. The cumulative incidences of MACEs, Re-MI, and any repeat revascularization were similar between the two groups (Table 2).

The condition of the STEMI group was worse than that of the NSTEMI group with respect to baseline characteristics. The values of cardiogenic shock $(5.2 \%$ vs. $1.6 \%, \mathrm{p}<0.001)$ and CPR on admission (2.9\% vs. $1.4 \%, \mathrm{p}<0.001)$; the number of current smokers $(48.6 \%$ vs. $38.5 \%, \mathrm{p}<0.001)$, LAD (IRA, treated vessel), RCA (IRA, treated vessel), and ACC/AHA type C; and the levels of CK-MB, troponin I were significantly higher in the STEMI group. However, the cumulative incidences of all-cause death and $C D$ were significantly lower in the STEMI group than in the NSTEMI group. These results were associated with the beneficial effects of RASI and were compatible with those of the OPTIMAAL study [21]. In the OPTIMAAL study, the clinical benefit of RASI was larger in the high-risk patient subgroup, including anterior MI, decreased LVEF ( $\leq 40 \%$ ), HF, prior MI, and tachycardia. On subgroup analysis, for patients who had decreased LVEF and who were in cardiogenic shock, statin combined with RASI reduced MACEs in patients with STEMI more than in those with NSTEMI (Fig. 3).
Another considerable factor for determining the cumulative incidences of all-cause death and $\mathrm{CD}$ was the treatment strategy. In the present study, $93.5 \%(11,627 / 12,490)$ of the STEMI patients had undergone primary PCI, and about $79.2 \%(7444 / 9400)$ of the NSTEMI patients had received early invasive treatment. The higher incidence of primary PCI may be associated with favorable all-cause death rates and CD in STEMI patients. Currently, the reasons for the higher incidence of death in NSTEMI during long-term follow-up remain poorly understood $[22,23]$. In patients with NSTEMI, studies recommended that a selective invasive strategy may be preferable in selected patients to improve long-term outcomes [24, 25].

KAMIR is a nationwide, prospective, observational, on-line registry in South Korea that has been compiling data since November 2005. More than 50 high-volume university and community hospitals with facilities for primary PCI and onsite cardiac surgery have participated [13]. Therefore, we believe the population of this study is sufficiently large to provide reasonably accurate results. Furthermore, the results of this comparative study may persuade interventional cardiologists of the benefits of statins combined with RASI with respect to reducing allcause death and CD in STEMI patients compared with those in NSTEMI patients after PCI.

\section{Limitations of the study}

Our study had several limitations. First, there may be some under-reporting and/or missed data because of the non-randomized retrospective nature of the study. Second, our study was based on medications at discharge, and the registry data did not include full detailed data concerning the starting time of statin or RASI therapy, changes in prescription doses, long-term adherence, or discontinuation during the follow-up period; therefore, these factors may contribute bias. Third, we could not provide serial follow-up results compared with initial laboratory results because of limitations related to the registry data; this too may introduce bias. Fourth, we reported 2-year clinical outcomes between the two groups in this study; nevertheless, a 2-year follow-up period is relatively short for the determination of long-term major clinical outcomes. Finally, the long inclusion period could also have influenced the patient's profile and may have biased the results, because RASI, AT1, and statins in recent years have been modernized in late generations with, generally, better bioprofile. 


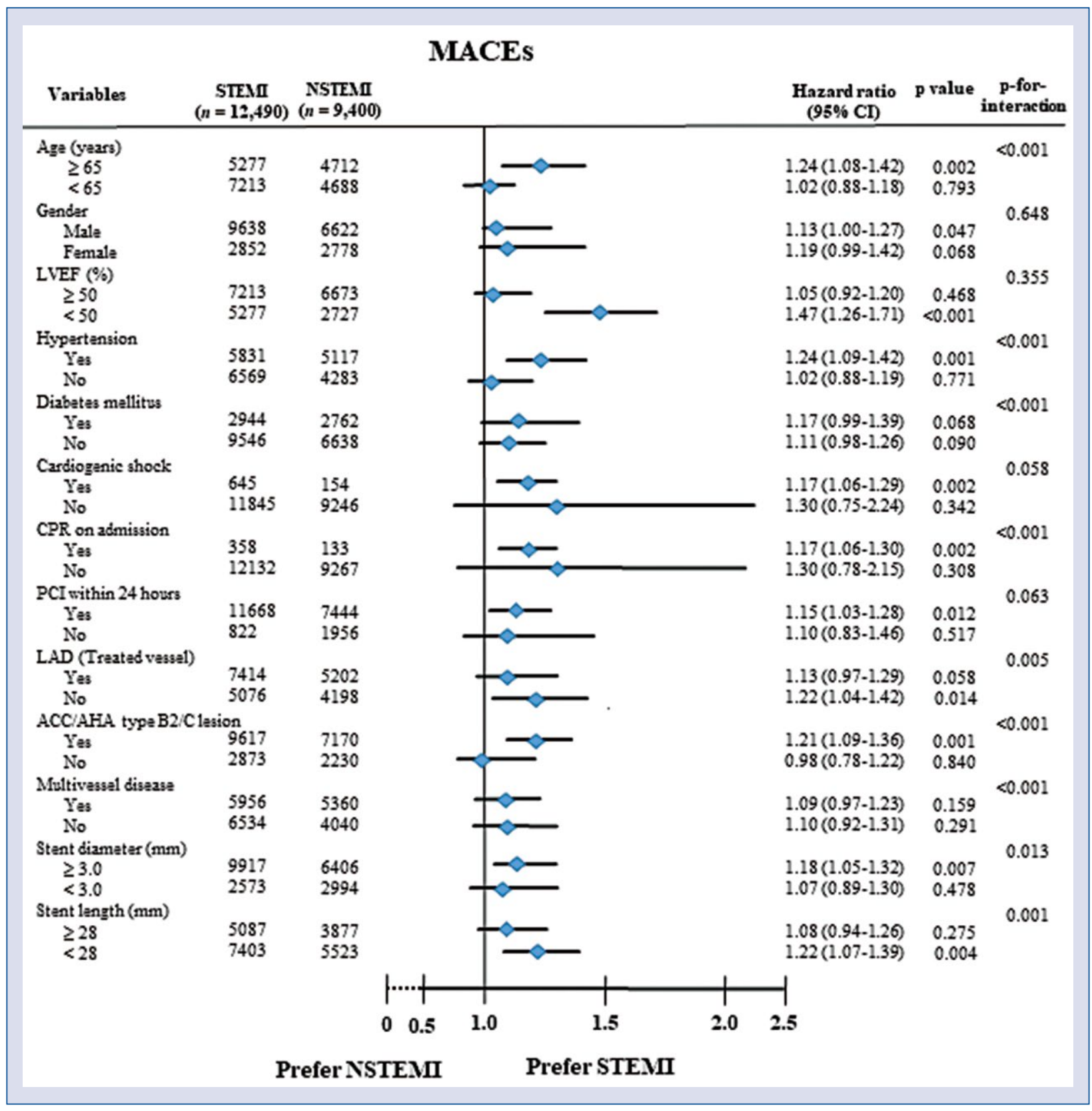

Figure 3. Subgroup analysis for major adverse cardiac events (MACEs); Cl — confidence interval; NSTEMI — non-ST-segment elevation myocardial infarction; STEMI — ST-segment elevation myocardial infarction; LVEF — left ventricular ejection fraction; $\mathrm{CPR}$ - cardiopulmonary resuscitation; $\mathrm{PCl}$ - percutaneous coronary intervention; LAD — left anterior descending artery; ACE/AHA - American College of Cardiology/American Heart Association.

\section{Conclusions}

In conclusion, despite the fact that the cumulative incidences of MACEs, Re-MI, and any repeat revascularization including TLR, TVR, and non-TVR were not statistically significantly different between the two groups, with respect to all-cause death and $\mathrm{CD}$ rates during a 2 -year follow-up period, combined use of statin with RASI was more effective in patients with STEMI than in those with NSTEMI. However, further studies are warranted to elucidate this focus.

\section{Acknowledgments}

This research was supported by funding (2016-ER6304-02) from the Research of Korea Centers for Disease Control and Prevention.

The authors thank the clinical investigators who contributed time and effort to this study, as 
well as the Korea Acute Myocardial Infarction Registry (KAMIR) investigators: Myung Ho Jeong, MD, Youngkeun Ahn, MD, Sung Chul Chae, MD, Jong Hyun Kim, MD, Seung-Ho Hur, MD, Young Jo Kim, MD, In Whan Seong, MD, Donghoon Choi, MD, Jei Keon Chae, MD, Taek Jong Hong, MD, Jae Young Rhew, MD, Doo-Il Kim, MD, In-Ho Chae, MD, Junghan Yoon, MD, Bon-Kwon Koo, MD, Byung-Ok Kim, MD, Myoung Yong Lee, MD, Kee-Sik Kim, MD, Jin-Yong Hwang, MD, Myeong Chan Cho, MD, Seok Kyu Oh, MD, Nae-Hee Lee, MD, Kyoung Tae Jeong, MD, Seung-Jea Tahk, MD, Jang-Ho Bae, MD, Seung-Woon Rha, MD, Keum-Soo Park, MD, Chong Jin Kim, MD, Kyoo-Rok Han, $\mathrm{MD}$, Tae Hoon Ahn, MD, Moo-Hyun Kim, MD, Ki Bae Seung, MD, Wook Sung Chung, MD, Ju-Young Yang, MD, Chong Yun Rhim, MD, Hyeon-Cheol Gwon, MD, Seong-Wook Park, MD, Young-Youp Koh, MD, Seung Jae Joo, MD, Soo-Joong Kim, MD, Dong Kyu Jin, MD, Jin Man Cho, MD, Sang-Wook Kim, MD, Jeong Kyung Kim, MD, Tae Ik Kim, MD, Deug Young Nah, MD, Si Hoon Park, MD, Sang Hyun Lee, MD, Seung Uk Lee, MD, Hang-Jae Chung, MD, Jang-Hyun Cho, MD, Seung Won Jin, MD, Myeong-Ki Hong, MD, Yangsoo Jang, MD, Jeong Gwan Cho, MD, Hyo-Soo Kim, MD, and Seung-Jung Park, MD.

\section{Conflict of interest: None declared}

\section{References}

1. Cannon $\mathrm{CP}$, Braunwald $\mathrm{E}, \mathrm{McCabe} \mathrm{CH}$, et al. Intensive versus moderate lipid lowering with statins after acute coronary syndromes. N Engl J Med. 2004; 350(15): 1495-1504, doi: 10.1056/ NEJMoa040583, indexed in Pubmed: 15007110.

2. Baigent C, Blackwell 1, Emberson J. Efficacy and safety of more intensive lowering of LDL cholesterol: a meta-analysis of data from 170000 participants in 26 randomised trials. Lancet. 2010; 376(9753): 1670-1681, doi: 10.1016/s0140-6736(10)61350-5.

3. Larsen AI, Tomey MI, Mehran R, et al. Comparison of outcomes in patients with ST-segment elevation myocardial infarction discharged on versus not on statin therapy (from the Harmonizing Outcomes With Revascularization and Stents in Acute Myocardial Infarction Trial). Am J Cardiol. 2014; 113(8): 1273-1279, doi: 10.1016/j.amjcard.2014.01.401, indexed in Pubmed: 24576541.

4. Piepoli MF, Hoes AW, Agewall S, et al. 2016 European Guidelines on cardiovascular disease prevention in clinical practice: The Sixth Joint Task Force of the European Society of Cardiology and Other Societies on Cardiovascular Disease Prevention in Clinical Practice (constituted by representatives of 10 societies and by invited experts)Developed with the special contribution of the European Association for Cardiovascular Prevention \& Rehabilitation (EACPR). Eur Heart J. 2016; 37(29): 2315-2381, doi: 10.1093/eurhearti/ehw106, indexed in Pubmed: 27222591.

5. Ibanez B, James S, Agewall S, et al. 2017 ESC Guidelines for the management of acute myocardial infarction in patients presen- ting with ST-segment elevation: The Task Force for the management of acute myocardial infarction in patients presenting with ST-segment elevation of the European Society of Cardiology (ESC). Eur Heart J. 2018; 39(2): 119-177, doi: 10.1093/eurheartj/ehx393, indexed in Pubmed: 28886621.

6. Amsterdam EA, Wenger NK, Brindis RG, et al. 2014 AHA/ACC Guideline for the management of patients with non-ST-elevation acute coronary syndromes: a report of the American College of Cardiology/American Heart Association Task Force on Practice Guidelines. J Am Coll Cardiol. 2014; 64(24): e139-e228, doi: 10.1016/j.jacc.2014.09.017, indexed in Pubmed: 25260718.

7. Kim YH, Her AY, Jeong MHo, et al. Different statin effects of ST-elevation versus non-ST-elevation acute myocardial infarction after stent implantation. Am J Med Sci. 2020; 359(3): 156-167, doi: 10.1016/j.amjms.2019.12.004, indexed in Pubmed: 32089157.

8. O'Gara PT, Kushner FG, Ascheim DD, et al. 2013 ACCF/AHA guideline for the management of ST-elevation myocardial infarction: a report of the American College of Cardiology Foundation/American Heart Association Task Force on Practice Guidelines. Circulation. 2013; 127(4): e362-e425, doi: 10.1161/ CIR.0b013e3182742cf6, indexed in Pubmed: 23247304.

9. Roffi M, Patrono C, Collet JP, et al. 2015 ESC Guidelines for the management of acute coronary syndromes in patients presenting without persistent ST-segment elevation: Task Force for the Management of Acute Coronary Syndromes in Patients Presenting without Persistent ST-Segment Elevation of the European Society of Cardiology (ESC). Eur Heart J. 2016; 37(3): 267-315, doi: 10.1093/eurheartj/ehv320, indexed in Pubmed: 26320110.

10. Kim YH, Her AY, Jeong MHo, et al. Impact of renin-angiotensin system inhibitors on long-term clinical outcomes in patients with acute myocardial infarction treated with successful percutaneous coronary intervention with drug-eluting stents: comparison between STEMI and NSTEMI. Atherosclerosis. 2019; 280: 166-173, doi: 10.1016/j.atherosclerosis.2018.11.030, indexed in Pubmed: 30529829.

11. Lee HY, Sakuma I, Ihm SH, et al. Statins and renin-angiotensin system inhibitor combination treatment to prevent cardiovascular disease. Circ J. 2014; 78(2): 281-287, doi: 10.1253/circj.cj-131494, indexed in Pubmed: 24401609.

12. Koh KK, Sakuma I, Hayashi T, et al. Renin-angiotensin system inhibitor and statins combination therapeutics: what have we learnt? Expert Opin Pharmacother. 2015; 16(7): 949-953, doi: 10.1517/14656566.2015.1019464, indexed in Pubmed: 25747324.

13. Kim Y, Ahn Y, Cho MC, et al. Current status of acute myocardial infarction in Korea. Korean J Intern Med. 2019; 34(1): 1-10, doi: 10.3904/kjim.2018.381, indexed in Pubmed: 30612415.

14. Kim YH, Her AY, Jeong MHo, et al. Two-year outcomes of statin therapy in patients with acute myocardial infarction with or without dyslipidemia after percutaneous coronary intervention in the era of new-generation drug-eluting stents within Korean population: Data from the Korea Acute Myocardial Infarction Registry. Catheter Cardiovasc Interv. 2019; 93(7): 1264-1275, doi: 10.1002/ccd.27985, indexed in Pubmed: 30474346.

15. Grech $\mathrm{ED}$. ABC of interventional cardiology: percutaneous coronary intervention. II: the procedure. BMJ. 2003; 326(7399): 1137-1140, doi: 10.1136/bmj.326.7399.1137, indexed in Pubmed: 12763994.

16. Won KB, Hur SH, Nam CW, et al. Evaluation of the impact of statin therapy on the obesity paradox in patients with acute myocardial infarction: A propensity score matching analysis from the 
Korea Acute Myocardial Infarction Registry. Medicine (Baltimore). 2017; 96(35): e7180, doi: 10.1097/MD.0000000000007180, indexed in Pubmed: 28858077.

17. Kim YH, Her AY, Jeong MH, et al. Clinical outcomes at 2 years between beta-blockade with ACE inhibitors or arbs in patients with AMI who underwent successful PCI with DES: a retrospective analysis of 23,978 patients in the korea AMI registry. Am J Cardiovasc Drugs. 2019; 19(4): 403-414, doi: 10.1007/s40256019-00326-8, indexed in Pubmed: 30788675.

18. Athyros VG, Kakafika AI, Tziomalos K, et al. Pleiotropic effects of statins: clinical evidence. Curr Pharm Des. 2009; 15(5): 479-489, doi: 10.2174/138161209787315729, indexed in Pubmed: 19199976.

19. Baigent C, Keech A, Kearney PM. Efficacy and safety of cholesterol-lowering treatment: prospective meta-analysis of data from 90056 participants in 14 randomised trials of statins. Lancet. 2005; 366(9493): 1267-1278, doi: 10.1016/s01406736(05)67394-1.

20. Koh KK, Han SH, Oh PC, et al. Combination therapy for treatment or prevention of atherosclerosis: focus on the lipid-RAAS interaction. Atherosclerosis. 2010; 209(2): 307-313, doi: 10.1016/j. atherosclerosis.2009.09.007, indexed in Pubmed: 19800624.

21. Dickstein K, Kjekshus J. Effects of losartan and captopril on mortality and morbidity in high-risk patients after acute myocar- dial infarction: the OPTIMAAL randomised trial. Lancet. 2002; 360(9335): 752-760, doi: 10.1016/s0140-6736(02)09895-1.

22. Hasdai D, Behar S, Wallentin L, et al. A prospective survey of the characteristics, treatments and outcomes of patients with acute coronary syndromes in Europe and the Mediterranean basin; the Euro Heart Survey of Acute Coronary Syndromes (Euro Heart Survey ACS). Eur Heart J. 2002; 23(15): 1190-1201, doi: 10.1053/euhj.2002.3193, indexed in Pubmed: 12127921.

23. Polonski L, Gasior M, Gierlotka M, et al. A comparison of ST elevation versus non-ST elevation myocardial infarction outcomes in a large registry database: are non-ST myocardial infarctions associated with worse long-term prognoses? Int J Cardiol. 2011; 152(1): 70-77, doi: 10.1016/j.ijcard.2010.07.008, indexed in Pubmed: 20684999.

24. Kofoed KF, Kelbæk H, Hansen PR, et al. Early versus standard care invasive examination and treatment of patients with non-ST-segment elevation acute coronary syndrome. Circulation. 2018; 138: 2741-2750, doi: 10.1161/CIRCULATIONAHA, indexed in Pubmed: 30565996.

25. Hoedemaker NPG, Damman P, Woudstra P, et al. Early invasive versus selective strategy for non-ST-Segment elevation acute coronary syndrome: the ICTUS trial. J Am Coll Cardiol. 2017; 69(15): 1883-1893, doi: 10.1016/j.jacc.2017.02.023, indexed in Pubmed: 28408018. 